\title{
Topology Characteristics of Resting-State Brain Network in Patients with Alzheimer's Disease
}

\author{
Ping $\mathrm{Hu}^{\mathrm{a}}$, Ting $\mathrm{Mei}^{\mathrm{b}, *}$ \\ School of Humanities and Information Management, Chengdu Medical College, Chengdu 610500, \\ China \\ awhoopingfwl@163.com, bmeiting@cuit.edu.cn
}

Keywords: Brain network, Patients with AD, Topology characteristics.

\begin{abstract}
To provide technical support for computer diagnosis of Alzheimer's disease (AD) patients, the data of resting-state functional magnetic resonance imaging (fMRI) are collected from 9 AD patients and 11 normal elderly people. We adopt the Automated Anatomical Labeling (AAL) template to divide the brain into 90 regions. The corresponding time series of each region are calculated and the values in the correlation matrix after mathematics transformation are taken as the weights of edges between the brain regions. Then we build the weighted brain network of each subject. Based on the above, we study the characteristics of the brain network in the specific brain regions of $\mathrm{AD}$ patients and normal elderly group respectively. The simulation results show that compared with the normal elderly group, the network efficiency of the weighted brain network in patients with $\mathrm{AD}$ decreased, and the characteristic path lengths are longer than those of the normal group under different Sparsity thresholds. This result can provide a theoretical basis for early diagnosis of $\mathrm{AD}$ patients.
\end{abstract}

\section{Introduction}

Alzheimer's disease, also known as senile dementia, is an insidious onset of neural degenerative diseases. Its clinical symptoms mainly include Gradual death of neurons progressive neuronal death, loss of memory, aphasia, decline of daily life ability and even brain death [1]. At present, Alzheimer's disease has become a serious public health problem all over the world. It brings not only great pain to patients and their families, but also a heavy burden to the society.

Complex network theory is a new method to study complex system rising in recent years. It can explore the relationship between system and network topology more deeply from the system level [2]. In fact, the human brain can also be abstracted as a complex network, and then we can use the method of complex network theory to analyze and investigate the structure and function of different brain regions [3,4]. Ref.[5] introduced the "small world" networks when dementia was studied, and found that the brain functional networks in patients with $\mathrm{AD}$ had longer characteristic path length longer than normal brain networks. According to the resting-state fMRI data of 5 normal subjects, Ref. [6] set up the corresponding brain networks of 5normal subjects. By calculating the characteristic path length and clustering coefficient of these two statistical characteristics, Ref. [6] found that these normal brain function networks had the characteristics of "small world". Ref. [7] showed that the hippocampal clustering coefficient of brain network in patients with AD decreased significantly, which could separate the AD patients from normal people accurately. This could greatly improve the efficiency of clinical AD diagnosis. Most researches above analyze the changes of the statistical characteristics of the brain functional networks in patients with $\mathrm{AD}$, but these brain networks are unweighted. Since the closeness between the brain regions can not be exactly the same, in order to accord with the fact, we will study the topology characteristics based on the weighted brain networks. Through the acquisition of resting-state fMRI data and the data preprocessing, we construct the weighted brain networks of patients with $\mathrm{AD}$ and normal elderly group. Then we compare the network statistical characteristics to analyze the network efficiency and characteristic path length of the weighted brain function network in patients with AD. 
The outline of this paper is as follows: in Section 2, we describe the establishment of the weighted brain network. We introduce the calculation process of the network efficiency and characteristic path length in Section 3. In Section 4, we make a case study. Finally, a summary and conclusions are stated in Section 5.

\section{Weighted Brain Network Establishment}

\subsection{FMRI Data Collection.}

In this study, a total of 20 subjects were collected, which included 9 elderly patients as AD group, 11 healthy volunteers as a control group. AD patients aged from 78 to 99 years old, and the ages of the control group ranged from 72 to 88. All patients with AD were diagnosed by clinical diagnosis. All subjects signed informed consent by himself or his guardian. The basic data of AD patients was shown in Table 1.

Table 1 Basic data of patients with AD

\begin{tabular}{cccccccccc}
\hline Number & 1 & 2 & 3 & 4 & 5 & 6 & 7 & 8 & 9 \\
\hline Gender & $\mathrm{M}$ & $\mathrm{M}$ & $\mathrm{F}$ & $\mathrm{F}$ & $\mathrm{M}$ & $\mathrm{M}$ & $\mathrm{M}$ & $\mathrm{F}$ & $\mathrm{F}$ \\
Age & 83 & 89 & 78 & 92 & 99 & 89 & 84 & 88 & 93 \\
\hline
\end{tabular}

In this experiment, fMRI was used to collect data, and the mode of brain function connectivity was researched in the resting state. Resting-state fMRI and structural image of all tested subjects were performed using a SIEMENS Signa 3.0T magnetic resonance scanner.

The main scanning parameters were set as follows. 35 axial slices, repetition time (TR) $=2000 \mathrm{ms,}$ echo time $(\mathrm{TE})=30 \mathrm{~ms}$, flip angle $(\mathrm{FA})=90^{\circ}$, thickness $=3.0 \mathrm{~mm}$, skip $=1 \mathrm{~mm}$, field of view $(\mathrm{FOV})=$ $200 \times 200 \mathrm{~mm}$, matrix $=64 \times 64,127$ volumes. During the experiment, all the subjects were closed, in stable mood, not moving around, without thinking activities but not sleeping, so the operation was relatively simple.

\subsection{Preprocessing of fMRI Data.}

In order to analyze data simply and accurately, we adopted SPM8 toolkits to preprocess fMRI data and analyze statistical parameters. Firstly, Corrected the image time. Secondly, removed the influence of parameters due to brain shaking on the map. Thirdly, standardized the scanned image into the Talairach space. And then smoothed the image using the $4 \times 4 \times 4$ Gauss filter core (FWHM) [8] to ensure a better signal to noise ratio (SNR). At last, removed the linear drift and filter for the map [9].

\subsection{Model Weighted Brain Network.}

The brain was divided into 90 brain regions by the Automated Anatomical Labeling (AAL), and each region represented a node. Calculated the average time series of each brain region. Subsequently, calculated Pearson's correlation coefficient between two regions, and then applied Fisher $r$ to $z$ transform. Finally we got a standard $90 \times 90$ correlation matrix $Z$. Took the sparsity in sparse network as the threshold, which was generally in the interval $[0.1,0.5]$. Compared each value in the matrix $Z$ with the threshold value. If the value in $Z$ was greater than the threshold value, there would be an edge between these two brain regions. Otherwise, there was no edge. In addition, if node $i$ connected node $j$, we defined the corresponding value in $Z$ as the weight of edge $(i, j)$. In this way, the weighted brain function network was constructed for the patients with AD and normal elderly group.

\section{Statistical Characteristics Analysis}

After construct the weighted brain function network in patients with AD and normal elderly group mentioned in Section 2, we use complex network theory to analyze the statistical characteristics of the weighted brain networks in two groups. In this paper, we choose two statistics, which are network efficiency centrality and characteristic path length respectively. Contrasting to weighted brain networks in normal elderly group, we explore the changes of topology characteristics of the weighted brain networks in patients with AD. 


\subsection{Network Efficiency Centrality.}

Efficiency centrality features how central a node is located in the network. Or to say, it can measure how easily a node gets to other nodes. It is not difficult to find the node with higher efficiency centrality implies a more central position in the global scope. The efficiency centrality of node $i$ is [10]:

$$
E C(i)=\frac{1}{n} \sum_{j=1, j \neq i}^{n} \frac{1}{\operatorname{dist}(i, j)}
$$

Where $\operatorname{dist}(i, j)$ represents the shortest path length from node $i$ to node $j . n$ is the network scale.

For the unweighted network, the shortest path length $\operatorname{dist}(i, j)$ between node $i$ and node $j$ is the minimum number of edges connecting the 2 nodes. But for the weighted network, dist $(i, j)$ is the minimum sum of all edge weights along the path from node $i$ to node $j$.

\subsection{Characteristic Path Length.}

Characteristic path length is a measure of the average distance between nodes in the network. It is also called the average path length. The characteristic path length $L$ of the network is the average value of the shortest path length between all nodes:

$$
L=\frac{2}{n(n+1)} \sum_{i \neq j=1}^{n} \operatorname{dist}(i, j)
$$

\section{Case Study}

In order to explore more fully the topology characteristics changes of the weighted brain networks in patients with $\mathrm{AD}$, we select different threshold values to construct different weighted brain networks. In view of the fact that the threshold value is generally in $[0.1,0.5]$, we set each increment of the threshold to 0.08 each time. After the construction of brain networks, we calculate the statistical characteristics of each tested subject, and then average the characteristics in their group. The average is the statistical characteristics of the group corresponding to the threshold. Therefore, for each group of subjects, there are 51 statistical-characteristic values corresponding to the 51 threshold points.

\subsection{Brain Network Efficiency}

Fig. 1 shows the changes in the weighted brain network efficiency of the patients with AD and the normal elderly group. In Fig.1, the blue square represents the efficiency of the weighted brain networks in AD group under different threshold values, and the red circle is that in normal elderly group under the same case. From Fig.1, we can see that the weighted brain network efficiency of AD patients is lower than that of the normal group, no matter which value the threshold is.

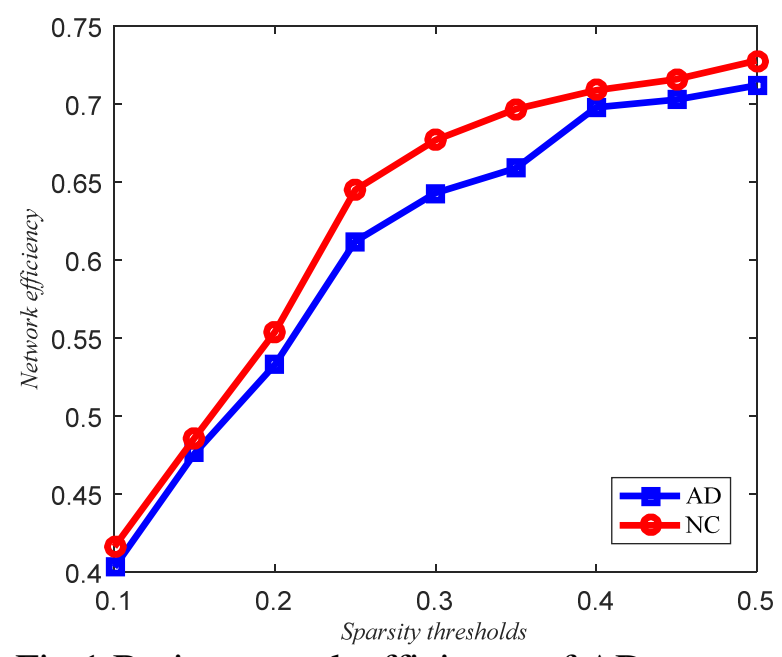

Fig.1 Brain network efficiency of AD group and normal elderly group

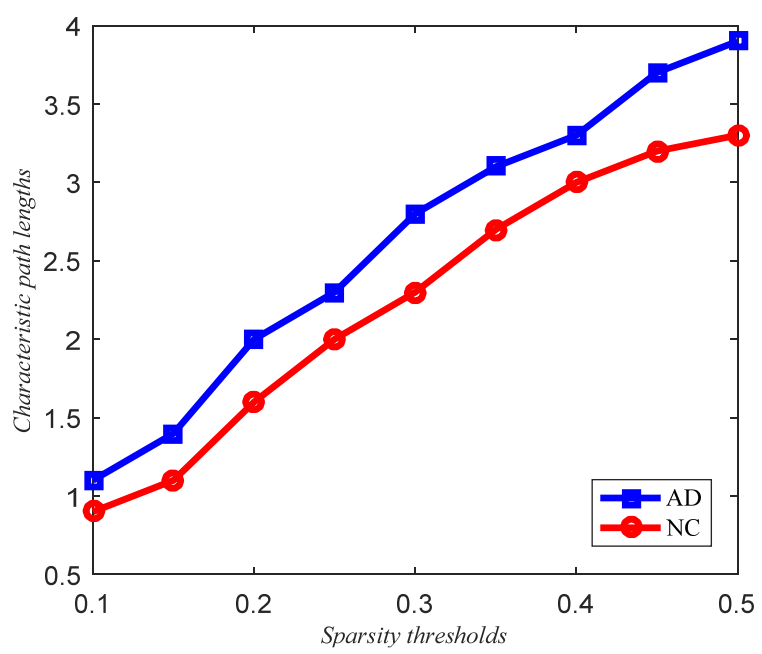

Fig.2 Characteristic path length of brain networks in $\mathrm{AD}$ group and normal elderly group 


\subsection{Characteristic Path Length of Brain networks}

Fig. 2 displays the characteristic path length of brain networks in AD group and normally elderly group. The blue square denotes the characteristic path length of the weighted brain networks in AD group under different threshold values, and the red circle represents the corresponding part in normal elderly group under different threshold values. Obviously, the blue curve is higher than the red curve. That is to say, the characteristic path lengths of AD patients are longer that those of normal people under different threshold values.

\section{Conclusion}

In this paper, we have first collected the fMRI data from the AD group and normal elderly group. And then we have built the weighted brain network for each tested subject after a series of data processing. We have compared and analyzed the statistical characteristics between AD group and normal elderly group. The results have shown that the patients with AD have lower brain network efficiency than the normal elderly people in the weighted-based brain networks under different threshold values. In addition, similarly based on the weighted brain networks, the characteristic path lengths of $\mathrm{AD}$ patients are longer that those of normal people under different threshold values. These indicate that the brains of patients with $\mathrm{AD}$ have lower speeds of information handling and transferring, which can also explain why the AD patients have poor cognitive ability. The research results in this paper are expected to provide some decision support for the early diagnosis of AD.

\section{References}

[1]. Ren M. The pathogenesis of Alzheimer's disease is expected to crack. Beijing Daily, 2014-7-4(5).

[2]. Newman M E J. The structure and function of complex networks. SIAM Review. Vol. 45 (2003) No. 2, p. 167-256.

[3]. Zhou P, Tian M, Zhang X, et al. Resting-State Brain Network Dysfunction Characteristic of Alzheimer's Disease. Nanotechnology and Precision Engineering. Vol. 13 (2015) No. 5, p. 346-352.

[4]. Feng L, Nie Z Y, Wang X B, et al. Resting fMRI evaluation of altered network efficiency in patients with Alzheimer Disease. Chinese Journal of Medical Imaging Technology. Vol. 29 (2013) No. 10, p. 1570-1574.

[5]. Stam C J, Jones B F, Nolte G, et al. Small-world networks and functional connectivity in Alzheimer's disease. Cerebral cortex. Vol. 17 (2007) No. 1, p. 92-99.

[6]. Salvador R, Suckling J, Coleman M R, et al. Neurophysiological architecture of functional magnetic resonance images of human brain. Cerebral cortex. Vol. 15 (2005) No.9, p. 1332-1342.

[7]. Supekar K, Menon V, Rubin D, et al. Network analysis of intrinsic functional brain connectivity in Alzheimer's disease[J]. PLoS Computer Biology. Vol. 4 (2008) No. 6, p. e1000100/1-11.

[8]. Chen H, Yang F, Huang Y, Chen J Q. Effects Study of SPM in the Functional Magnetic Resonance Imaging (fMRI) of Needling at Waiguan (TE5). Chinese Journal of Medical Physics. Vol. 28 (2011) No.1, p. 2369-2372.

[9]. Li Y P, Qin Y Y, Li W, et al. The Functional Brain Network Changes of Alzheimer's Disease. Chinese Journal of Medical Physics. Vol. 30 (2013) No.6, p. 4510-4514.

[10]. Zhou X, Zhang F M, Li K W, et al. Finding vital node by node importance evaluation matrix in complex networks[J]. Acta Physica Sinica. Vol. 61 (2012) No.5, p.050201/1-5. 